\title{
Europaarbeit der Gewerkschaft ver.di. Vorschlag einer feldtheoretischen Perspektive
}

\author{
Nele Dittmar
}

(C) Der/die Autor(en) 2017. Dieser Artikel ist eine Open-Access-Publikation.

Zusammenfassung In diesem Artikel werden erste Vorschläge dazu gemacht, wie die Europaarbeit der deutschen Gewerkschaft ver.di aus feldtheoretischer Perspektive betrachtet werden kann. Die Organisation ver.di wird dabei selbst als ein Feld begriffen. Es wird dargestellt, welche Akteur/innen in ver.di mit europäischen Themen befasst sind und welche Machtrelationen zwischen ihnen bestehen. Diese ergeben sich durch die Ausstattung mit feldspezifischem Kapital. Es zeigt sich, dass die Europapolitik in ver.di sehr dezentral organisiert ist und den einzelnen Fachbereichen, die über viel feldspezifisches Kapital verfügen, eine wichtige Rolle zukommt. Das EU-Verbindungsbüro ver.dis nimmt demgegenüber in der Organisation-als-Feld eine eher dominierte Position ein. Dies führt dazu, dass die Europapolitik ver.dis nur in geringem Maße zentral koordiniert wird.

Schlüsselwörter Gewerkschaft · Europa $\cdot$ Feldtheorie $\cdot$ Organisation-als-Feld

\section{European policy of the union ver.di. Proposal of a field theoretical perspective}

\begin{abstract}
This article offers some suggestions on how to look at the organization of Europe-related work within the German union ver.di from a field theoretical perspective. The organization ver.di is itself conceptualized as a field. The article describes which actors in ver.di are involved in European policy making and sketches the power relations between these actors. These are defined by the endowment with field-specific capital. It becomes apparent that European policy is organized very decentrally within ver.di and that the different sections in ver.di that possess a lot of field-specific capital play an important role. The EU-liaison office in ver.di on the
\end{abstract}

N. Dittmar $(\bowtie)$

Altenberger Straße 69, 4040 Linz, Österreich

E-Mail: nele.dittmar@jku.at 
other hand holds a rather dominated position within the organization-as-field. As a consequence, there is only little central coordination of ver.di's European policy.

Keywords Trade union $\cdot$ Europe $\cdot$ Field theory $\cdot$ Organization-as-field

\section{Einleitung}

Zahlreiche Hürden, die transnationalem Handeln von Gewerkschaften in Europa entgegenstehen, sind in der Literatur ausführlich beschrieben worden (z. B. Bernaciak 2013; Greer und Hauptmeier 2012; Pernicka und Glassner 2014). Seltener wurde aber bisher in eine nationale Gewerkschaft hineingeschaut, um zu untersuchen, ob und inwiefern die innergewerkschaftlichen (Macht-)Verhältnisse ihre europabezogene Arbeit beeinflussen (s. als eines der wenigen Beispiele Rüb 2009 zur IG Metall). Eine solche Perspektive könnte allerdings einerseits zu einem besseren Verständnis der Schwierigkeiten transnationalen Handelns von Gewerkschaften beitragen, andererseits jedoch auch vorhandene europabezogene Praktiken zum Vorschein bringen, die bei einem auf die „Makroebene“ beschränkten Blick verdeckt bleiben. Ein solcher Blick ins Innere einer nationalen Gewerkschaft soll hier am Beispiel der deutschen Vereinten Dienstleistungsgewerkschaft ver.di versucht werden. Ver.di ist die größte Dienstleistungsgewerkschaft in Europa und spielt entsprechend eine bedeutende Rolle für die europäischen Gewerkschaftsdachverbände. Daher ist eine Untersuchung dieser Organisation auch über den Einzelfall hinaus von Interesse.

Für den beabsichtigten Blick ins Innere der Gewerkschaft wird hier eine feldtheoretische Perspektive vorgeschlagen, um damit ,die ungleiche Verteilung von Machtressourcen (,Kapitalsorten') als ein konstituierendes Element der sozialen Genese organisatorischer Strukturen und Strategien“ ins Zentrum der Betrachtung zu rücken (Dederichs und Florian 2002, S. 77). Mit Bourdieu ist ,ein Feld als ein Netz oder eine Konfiguration von objektiven Relationen zwischen Positionen“ (Bourdieu und Wacquant 1996, S. 127) zu sehen, wobei die Positionen und ihre Relationen zueinander durch die Struktur der Kapitalverteilung innerhalb des Feldes bestimmt werden (Bourdieu und Wacquant 1996, S. 127). Welche Kapitalsorten in einem Feld relevant sind, muss empirisch bestimmt werden. Feldspezifisches Kapital ist das, was in dem betreffenden Feld auf dem Spiel steht und was es seinem Besitzer oder seiner Besitzerin erlaubt, in diesem Feld überhaupt Einfluss zu nehmen (Bourdieu und Wacquant 1996, S. 128). Aus einer feldtheoretischen Perspektive muss man davon ausgehen, dass auch eine Organisation in einem Feld selbst keine einheitliche Akteurin ist, sondern wiederum als Feld begriffen werden muss (Bourdieu 2005, S. 205). Nicht „die Organisation“ trifft eine Entscheidung, verfolgt eine europapolitische Strategie oder hat sich bestimmte europabezogene Praktiken angeeignet. Vielmehr kann eine Organisation als ein ,„mesosoziale[r]' Zusammenhang“ begriffen werden, „,der durch die sozialen Kräfteverhältnisse und mikropolitischen Auseinandersetzungen der in Bereiche und Abteilungen, Berufs- und Arbeitsgruppen differenzierten ,Mitglieder" hergestellt wird" (Dederichs und Florian 2002, S. 89 f.). In feldtheoretischer Perspektive laufen in einer Organisation permanent Auseinandersetzungen und Aushandlungsprozesse um Regeln, Strukturen und Strategien ab, die von Ak- 
teurInnen ausgetragen werden, die mit unterschiedlichem und unterschiedlich viel Kapital ausgestattet sind und ihre Anliegen daher besser oder schlechter gegenüber anderen durchsetzen können (Dederichs und Florian 2002, S. 77). Dabei ist es wichtig zu beachten, dass die formale Organisationsstruktur nicht der faktisch wirksamen Machtstruktur in der Organisation entsprechen muss. Dies ist damit zu erklären, dass „formales Kapital“ oder das Kapital, das mit der Einnahme einer Position in der formalen Organisationsstruktur verbunden ist, nur eine der Kapitalformen ist, die im Feld der Organisation zählen können. Darüber hinaus können durchaus noch andere Kapitalsorten in der Organisation wirksam sein (Emirbayer und Johnson 2008, S. 23). Welchen Arten von Kapital in einer Organisation besonderer Wert beigemessen wird, ist das Ergebnis von Auseinandersetzungen im organisationsinternen Feld der Macht (Emirbayer und Johnson 2008, S. 25). Obwohl davon ausgegangen wird, dass ein Feld nach eigenen Spielregeln ,funktioniert“, kann eine Organisation-als-Feld nicht unabhängig von ihrer Umwelt analysiert werden, durch die es zwar nicht determiniert, aber doch beeinflusst wird. Die Deutung und Perzeption externer Zwänge im Feld hängt davon ab, welche Deutungen in den Macht- und Herrschaftsverhältnissen im Feld durchgesetzt werden (können) (Hofbauer 2012, S. $437 \mathrm{f}.)^{1}$

In dieser Forschungsnotiz sollen nun erste Vorschläge und Überlegungen für eine feldtheoretische Betrachtungsweise der Europaarbeit ver.dis präsentiert werden. Diese sind im Rahmen eines Dissertationsprojektes entstanden und bedürfen der weiteren Ausarbeitung, um dem Vorhaben, eine Organisation als Feld zu analysieren, tatsächlich gerecht werden zu können. Zunächst sollen ver.dis Organisationsstruktur und die Verteilung der „Europakompetenzen“ skizziert werden. Um die Heterogenität der Europaarbeit ver.dis zu illustrieren, sollen danach kurz drei Fachbereiche porträtiert werden, die unterschiedlichen Branchenbedingungen ausgesetzt sind und die sich besonders stark in ihrer Betroffenheit von „Europa“ und ihrer europäischen Arbeit unterscheiden. Danach werden die Position des EU-Verbindungsbüros innerhalb ver.dis und seine Beziehungen zu den Fachbereichen genauer in den Blick genommen. Es folgt eine kurze Schlussbetrachtung. Die vorgestellten Ergebnisse basieren auf 12 ExpertInnen-Interviews mit VertreterInnen der Gewerkschaft ver.di aus Fach- und Querschnittsbereichen, die zwischen Februar 2013 und Juni 2015 geführt wurden, sowie den Erfahrungen im Rahmen eines Praktikums bei ver.di. Meine Untersuchung beschränkt sich auf die Ebene der ver.di-Bundesverwaltung.

\footnotetext{
1 Neben den Begriffen „Feld“ und „Kapital“ ist „Habitus“ das dritte wichtige Konzept bei Bourdieu. Welche Rolle unterschiedliche Habitus bei der Verfolgung europabezogener Praktiken in ver.di spielen könnten, kann hier aus Platzgründen nicht beleuchtet werden. Vaughan (2008, S. 67) geht aber davon aus, dass auch eine nur partielle Anwendung von Bourdieus Konzept sinnvoll sein kann.
} 


\section{Organisationsstruktur und Europakompetenzen in ver.di}

\section{1 ver.di als Feld}

ver.di entstand 2001 durch den Zusammenschluss von fünf Einzelgewerkschaften. ${ }^{2}$ Die Gewerkschaft organisiert 2,04 Mio. Mitglieder (Stand 2014) in einer Vielzahl von Branchen. Sie ist in 13 Fachbereiche und drei bzw. vier Ebenen gegliedert (Bundes-, Landesbezirks- und Bezirksebene sowie ggf. eine örtliche Ebene ${ }^{3}$ ).

Diese „Matrixstruktur“ ver.dis lässt den Fachbereichen und Ebenen bewusst ein recht hohes $\mathrm{Ma} ß$ an Eigenständigkeit und gibt den ebenfalls vorhandenen Querschnittsbereichen (wie z. B. der tarifpolitischen Grundsatzabteilung, dem Bereich Mitbestimmung und dem EU-Verbindungsbüro) nur geringe Steuerungsmöglichkeiten. Nach Einschätzung eines Querschnittsbereichsvertreters ist die Matrixstruktur bei der ver.di-Gründung nicht gewählt worden, weil sie für eine besonders effektive Organisationsform gehalten wurde, sondern sie sei Teil des „Gründungskompromisses“ gewesen. Die besondere Autonomie der Fachbereiche sei insbesondere vereinbart worden, um einer zu großen Dominanz der mitgliederstarken ÖTV vorzubeugen. Aus Sicht eines Vertreters eines Querschnittsbereichs stellt sich mittlerweile allerdings eher das Problem, dass „Fliehkräfte“ wirken, die die Differenzierung der Organisation noch verstärken (Interview 07).

Ich möchte in dieser Forschungsnotiz versuchsweise die Möglichkeit, innerhalb des eigenen Bereichs Mitglieder gewinnen oder halten zu können (,Mitgliederzugriff“), als eine wichtige Kapitalsorte innerhalb ver.dis begreifen. Darüber hinaus dürften sowohl das oben angesprochene ,formale Kapital“" sowie weitere feldspezifische Kapitalsorten von Bedeutung sein, die aber zu diesem Zeitpunkt der Untersuchung noch nicht systematisiert werden können. Obwohl die hier präsentierte Skizze der Machtverhältnisse innerhalb ver.dis durch die Konzentration auf die Kapitalsorte „Mitgliederzugriff“ eindimensional und unterkomplex bleibt, ermöglicht sie eine erste Annäherung an eine Betrachtung der Organisation ver.di als Feld.

Dem Gewinnen und Halten von Mitgliedern kommt in ver.di ein hoher Stellenwert zu, da ver.di ausschließlich über Mitgliedsbeiträge finanziert ist und diese zum „Überleben“ der Organisation als notwendig betrachtet werden. Nach in ver.di verbreiteter Auffassung (und belegt durch die Eintrittszahlen) werden Mitglieder insbesondere im Rahmen von Tarifauseinandersetzungen gewonnen. Den 13 Fachbereichen in ver.di, denen laut Satzung sowohl die Aufgabe der Mitgliederwerbung als auch die der operativen, fachbezogenen Tarifpolitik zukommt, wird aufgrund dessen nach dem Empfinden meiner InterviewpartnerInnen innerhalb der Organisation eine höhere Bedeutung beigemessen als den Querschnittsbereichen, die diesen Zugang zu den Mitgliedern nicht haben.

\footnotetext{
2 Die fünf ver.di-Gründungsgewerkschaften waren die Deutsche Angestellten-Gewerkschaft (DAG), die Deutsche Postgewerkschaft (DPG), die Gewerkschaft Handel, Banken und Versicherungen (HBV), die IG Medien und die Gewerkschaft Öffentliche Dienste, Transport und Verkehr (ÖTV).

3 Laut ver.di-Satzung ( $\$ 24)$ kann auf Initiative betroffener Mitglieder vom Bezirksvorstand über die Bildung von Ortsvereinen entschieden werden, wenn auf Ortsebene mehrere Fachbereiche bestehen.
} 
Die Mitgliederzahlen eines Fachbereichs bzw. die durch die Mitglieder gezahlten Beiträge sind maßgeblich für seine Ressourcenausstattung, womit der ,Mitgliederzugriff“ in ökonomisches Kapital konvertiert wird. Zwar sehe die Budgetierungsrichtlinie von ver.di eine gewisse Umverteilung zwischen den Bereichen vor, trotzdem fließe viel Geld in Bereiche, in denen der Organisationsgrad bereits hoch ist und gewerkschaftliche Strukturen bereits etabliert ,und entsprechend auch innergewerkschaftliche Machtpositionen vorhanden sind“ (Kempe 2011, S. 61). Durch den ,Mitgliederzugriff“ unterscheidet sich also nicht nur die Kapitalausstattung zwischen Fachbereichen und Querschnittsbereichen, sondern auch zwischen den Fachbereichen untereinander, wobei diejenigen Fachbereiche im Vorteil sind, in deren Branchen bereits gewerkschaftliche und tarifliche Strukturen verankert sind. Nach Einschätzung von Kempe hat die Budgetierungsrichtlinie zwar Verteilungskonflikte befriedet, lässt aber kaum Spielraum für flexiblen Mitteleinsatz (Kempe 2011, S. 62). Einer Veränderung der entsprechenden Regeln stehe die „organisationspolitisch[e] Immobilität und Selbstbeschäftigung in ver.di“ in Verbindung mit der Matrixstruktur entgegen ebenso wie ,durch die Mechanismen der Budgetierungsrichtlinie gestützten Bereichsegoismen“ (Kempe 2011, S. 102). Wie ein Fachbereichsvertreter berichtet, wird allerdings im Rahmen des derzeitigen Organisationsentwicklungsprogramms „Perspektive 2015“ geplant, der Mitgliedergewinnung in bisher schlecht erschlossenen Bereichen innerhalb von ver.dis Organisationsbereich eine höhere Priorität zu geben und auch die Ressourcenvergabe innerhalb ver.dis stärker danach auszurichten. Dies könnte nach Einschätzung des Fachbereichsvertreters die Logik der Mittelvergabe zu Ungunsten bereits gut organisierter Bereiche, deren Interesse in der Erhaltung dieses hohen Organisationsgrades liegt, verändern (Interview 06). Die Querschnittsabteilungen ohne Mitgliederanbindung würden allerdings auch durch die veränderte Logik nicht stärker begünstigt. Die derzeitige Maxime des Bundesvorstands - der wahrscheinlich als das organisationsinterne Feld der Macht begriffen werden kann ${ }^{4}$ - laute, ,je mitgliedernäher, desto begründeter ist die Verteilung von Ressourcen“ (Interview 07). Nach Auffassung eines Querschnittsbereichsvertreters werde die Zuarbeit der Querschnittsbereiche anhand ihres Nutzens für das Ziel der Mitgliedergewinnung bewertet. Bereiche, deren Arbeit als diesbezüglich eher irrelevant eingeschätzt werde, müssten ,eher um ihre Budgets kämpfen“ (Interview 07).

\subsection{Europakompetenzen in ver.di}

Die „Europakompetenzen“ in ver.di sind ihrer Organisationsform entsprechend nicht zentral gebündelt. Viele AkteurInnen innerhalb ver.dis sind europabezogen aktiv. Auf Ebene des Bundesvorstandes halten sowohl der ver.di-Vorsitzende als auch weitere Vorstandsmitglieder Mandate in den Vorständen der europäischen Gewerkschaftsver-

\footnotetext{
4 Nach Auffassung einiger InterviewpartnerInnen ist allein der ver.di-Bundesvorstand ein effektives, fachbereichsübergreifendes Steuerungs- bzw. Abstimmungsorgan (Interviews 03, 07). Dem Bundesvorstand gehören der Vorsitzende und 13 weitere Personen an. In den Zuständigkeitsbereichen (Ressorts) der Bundesvorstandsmitglieder befindet sich in den meisten Fällen ein Fachbereich.
} 
bände, in denen ver.di Mitglied ist. ${ }^{5}$ Auch viele der Querschnittsbereiche ver.dis sind mit europäischen Themen befasst. So ist der Bereich Mitbestimmung gemeinsam mit den Fachbereichen für die Unterstützung und Beratung Europäischer Betriebsräte (EBR) zuständig, und die tarifpolitische Grundsatzabteilung begleitet die europäische tarifpolitische Koordinierung, um nur zwei Beispiele zu nennen. Daneben gibt es als explizit für „Europa“ zuständige Stelle in ver.di das EU-Verbindungsbüro. Bei ver.di-Gründung existierte ein Bereich „Europapolitik“ und zusätzlich eine kleinere Abteilung für internationale Politik. Später wurde diese in den Europabereich integriert, woraufhin die internationale Arbeit erheblich reduziert wurde. Im Laufe der Zeit kam es im Bereich Europa und Internationales zu einem Abbau von personeller Kapazität, da Stellen von ausscheidenden KollegInnen nicht wieder besetzt wurden (Interview 02). Einige Aufgabenfelder, die zunächst auch im Bereich Europapolitik bearbeitet worden waren, gingen an andere Bereiche über bzw. es wurden Doppelzuständigkeiten beseitigt (so z. B. bei der EBR-Arbeit). Mittlerweile gibt es in ver.di keine offizielle Zuständigkeit für das Thema „Internationales“ außerhalb der Fachbereiche mehr, und aus dem Bereich „Europapolitik“ wurde das EU-Verbindungsbüro. Damit ging eine klare Fokusverschiebung in Richtung Lobbyarbeit bei den europäischen Institutionen einher, wobei diese Fokussierung in starkem Maße auch durch die zunehmende Entwicklung der europäischen Institutionen zu für ver.di relevanten Rechtsetzungsinstanzen begründet ist.

Die Fachbereiche verfügen auch beim Thema „Europa“ über eine relativ große Handlungsautonomie. Sie entscheiden selbst, wie viel Zeit und Ressourcen sie für die europäische Arbeit verwenden. Dies hängt auch in starkem Maße mit den Branchen zusammen, die ihren jeweiligen Organisationsbereich darstellen (z. B. besteht je nach Branche eine unterschiedliche Bedeutung von europäischen Regelungen oder auch multinationalen Unternehmen) sowie von ihrer Ressourcenausstattung bzw. personellen Kapazitäten und den Interessenlagen ihrer Mitglieder (Interviews 01, 02). So ergeben sich zwischen den Fachbereichen große Unterschiede bzgl. ihrer Europapolitik. In sehr unterschiedlicher Ausprägung und Intensität beteiligen sie sich an europäischer tarifpolitischer Koordinierung und am europäischen Sozialen Dialog, arbeiten in den jeweiligen europäischen Gewerkschaftsdachverbänden mit, betreiben Lobbying beim Europäischen Parlament (EP), betreuen EBR, bilden unternehmensbezogene Gewerkschaftsallianzen und informieren ihre Mitglieder zu europäischen Themen. Dies soll im Folgenden anhand von drei Beispielen illustriert werden.

\subsection{Kurzporträt der Europaarbeit von drei ver.di-Fachbereichen}

Der Fachbereich Verkehr umfasst Branchen, die stark internationalisiert, intensivem Wettbewerb und Liberalisierung ausgesetzt und von (De-)Regulierung durch die europäische und internationale Ebene betroffen sind. Aufgrund dessen stellen europäische bzw. internationale Themen im Fachbereich Verkehr anders als in anderen Bereichen einen integralen Bestandteil der alltäglichen Gewerkschaftsarbeit

\footnotetext{
5 Die drei größten sind UNI Europa (der europäische Teil des Dienstleistungsgewerkschaftsverbandes UNI Global Union), EGÖD (Europäischer Gewerkschaftsverband für den öffentlichen Dienst) und ETF (Europäische Transportarbeiter-Föderation).
} 
dar (Interviews 06, 08, 10). Während in anderen Fachbereichen die Beschäftigung mit europäischen Themen einen zusätzlichen Aufwand bedeutet, lässt sich wegen der starken Durchdringung der Branche von europäischen und internationalen Regelungen und darauf gerichteter Gewerkschaftsarbeit im europäischen und nationalen Rahmen im Fachbereich Verkehr die europäische Arbeit von der nationalen häufig kaum trennen. In einigen der Fachgruppen bestehen tägliche Kontakte zur ETF (in anderen zumindest wöchentlich), und es erfolgt eine aktive politikgestaltende Mitwirkung (Branchen- sowie Verbandspolitik) in den europäischen und internationalen Verbänden, wo die ver.di-SekretärInnen verschiedene Ämter bekleiden. Auslandsreisen der SekretärInnen sind keine Seltenheit. Der Verkehrsbereich nimmt im Vergleich der ver.di-Fachbereiche an den meisten sektoralen Sozialdialogen auf EUEbene teil. ${ }^{6}$ Es bestehen Kontakte zu Abgeordneten des EP, und es wird Lobbyarbeit betrieben. Im Bereich der Seeschifffahrt finden darüber hinaus Tarifverhandlungen auf internationaler Ebene statt (vgl. dazu z. B. auch Anner et al. 2006). Damit handele es sich im Verkehrsbereich um ,wirklich gelebte internationale Arbeit“ (Interview 06). Bereits in der ÖTV, die die Hauptquellgewerkschaft für den Fachbereich Verkehr in ver.di darstellt, hat der Verkehrsbereich seine europäische und internationale Arbeit intensiv und sehr eigenständig betrieben (Interview 12). Dies setzte sich innerhalb von ver.di fort. Während das EU-Verbindungsbüro in den europäischen Gewerkschaftsdachverbänden UNI Europa und EGÖD insbesondere in Bezug auf sektorübergreifende Themen neben den Fachbereichen intensiv mitarbeitet, erfolgt die Mitarbeit in der ETF weitgehend allein durch den Verkehrsbereich.

Die Kernbereiche des öffentlichen Dienstes sind in ver.di in zwei Fachbereichen organisiert („Bund und Länder“ und „Gemeinden“), die eng zusammenarbeiten. Im Bereich des öffentlichen Dienstes gibt es wenige direkte Regelungskompetenzen der EU, dennoch ist der öffentliche Sektor von europäischer Politik, wie beispielsweise der Fiskalpolitik oder Liberalisierungsvorhaben, durchaus betroffen. Anders als im Fachbereich Verkehr ist „Europa“ aber kein integraler Bestandteil der täglichen Arbeit, sondern wird als zusätzliche Aufgabe wahrgenommen, die neben dem Alltagsgeschäft bewältigt werden muss (z. B. die Teilnahme an Umfragen unter den Mitgliedsgewerkschaften seitens des EGÖD). Aufgrund der ohnehin knappen (Zeit-)Ressourcen und hohen Arbeitsbelastung wird der europäischen Arbeit daher nicht der Stellenwert zuteil, den sie nach Meinung der FachbereichsvertreterInnen eigentlich haben müsste. Verpflichtungen und Veranstaltungen ,im nationalen Rahmen", wie Tarifverhandlungen oder wichtige Konferenzen, haben eindeutigen Vorrang vor der Beteiligung oder Terminen in europäischen Kontexten (wie z. B. Terminen im Rahmen sektoraler Sozialdialoge, an denen beide Fachbereiche beteiligt sind) (Interview 05). Den europäischen Verpflichtungen in solchen Situationen den Vorrang zu geben „würde so auch nicht akzeptiert“ (Interview 11) werden. Im Gegensatz zum Verkehrsbereich, wo die Mitarbeit in den Zusammenhängen der europäischen Gewerkschaftsverbände etwas fast Alltägliches ist, mit dem sich die zuständigen SekretärInnen zum großen Teil vertraut fühlen, scheint die europäische Arbeit in den Fachbereichen des öffentlichen Dienstes sich in eher „formalisierten“

\footnotetext{
${ }^{6}$ Derzeit nimmt der Verkehrsbereich - teils in Zusammenarbeit mit anderen Gewerkschaften - an sechs sektoralen Sozialdialogen teil.
} 
Bahnen zu bewegen (z. B. Halten von Mandaten in Ausschüssen der europäischen Gewerkschaftsverbände).

Es zeigt sich, dass die heterogene Organisation ver.di in unterschiedliche Felder eingebettet ist und die dortigen Verhältnisse Einfluss auf die Gewerkschaft haben, ihre Politik aber nicht determinieren. Europäische Themen erfahren in ver.di insbesondere dann Aufmerksamkeit, wenn sie sich mit der herrschenden Organisationslogik in Einklang bringen lassen, das heißt dann, wenn europäische Regelungen relativ direkte Folgen für die nationale Tarifpolitik haben und wenn ,trotz“ der Befassung mit europäischen Themen Mitgliedernähe gewährleistet ist, weil diese selbst davon betroffen sind.

\section{Die Position des EU-Verbindungsbüros im Feld ,ver.di““}

Das EU-Verbindungsbüro in ver.di ist mit zwei GewerkschaftssekretärInnen und einer/-m MitarbeiterIn besetzt. Aufgabe der SekretärInnen ist es, Entwicklungen in der europäischen Politik und Rechtsetzungsvorhaben $\mathrm{zu}$ verfolgen, für ver.di relevante Informationen zu sammeln und an die betroffenen (Fach-)Bereiche weiterzuleiten sowie im Gegenzug ver.di-Positionen zu aggregieren bzw. bei übergreifenden Themen selbst zu erarbeiten und auf die europäische Ebene zurückzuspielen. Das EUVerbindungsbüro sieht sich selbst in einer „Scharnierfunktion“ zwischen Brüssel und den ver.di-Fachbereichen.

Das EU-Verbindungsbüro ist auf die Mitarbeit der Fachbereiche angewiesen, um fachliche Informationen zu den auf europäischer Ebene behandelten Themen zu bekommen. Der Zugang zu den Bereichen stellt sich allerdings zuweilen nicht einfach dar (Interview 01). Seitens der oben kurz vorgestellten Fachbereiche des öffentlichen Dienstes werden die Zusammenarbeit mit dem EU-Verbindungsbüro und der Informationsfluss aber als gut empfunden. Mit dem Verbindungsbüro wird insbesondere dann kooperiert, wenn es um Themen geht, zu denen sich Gesamt-ver.di positionieren muss. Es wird auch klar erwartet, dass das EU-Verbindungsbüro in Bezug auf ver.dis Europapolitik ,die großen Fäden (...) ziehen“ solle (Interview 05). Auch vom Verkehrsbereich werden die europäischen Kontakte sowie die Expertise des EU-Verbindungsbüros geschätzt, jedoch wird von einem/-r FachgruppenvertreterIn - selbstkritisch - festgestellt, dass die Potenziale einer engeren Zusammenarbeit derzeit noch nicht ausgeschöpft werden (Interview 06) bzw. in manchen Fällen die Rollenverteilung noch unklar sei (Interview 08). Die Häufigkeit, mit der Kontakte zum EU-Verbindungsbüro bestehen, scheint sich zwischen den einzelnen Fachgruppen im Verkehrsbereich aber zu unterscheiden.

Während der Informationsfluss vom Verbindungsbüro zu den Fachbereichen von den meisten FachbereichsvertreterInnen also positiv bewertet wird, ist das Verbindungsbüro über die eigenständige Europaarbeit der Fachbereiche allerdings nicht systematisch informiert. Es ist beispielsweise nicht immer bekannt, welche KollegInnen an welchen Ausschüssen oder Arbeitsgruppen in den europäischen Gewerkschaftsverbänden teilnehmen, welche Sozialen Dialoge durch ver.di besetzt sind oder wie die Fachbereiche intern ihre Europaarbeit organisieren (Interview 01). Darüber hinaus werden auch die vielfältigen Kontakte zu AkteurInnen in Brüssel, über 
die sowohl die Fachbereiche als auch das EU-Verbindungsbüro verfügen, in ver.di nicht gebündelt oder abgestimmt.

Die „Diskussionsgruppe Europa“, zu der das EU-Verbindungsbüro die Europazuständigen aus allen (Fach-)Bereichen ver.dis viermal im Jahr zur Diskussion eher breiterer Themen einlädt, stehe in der Priorität hinter dem (tarifpolitischen) Alltagsgeschäft der KollegInnen zurück (Interview 01). An der Diskussionsgruppe nehmen meist deutlich mehr KollegInnen aus den ver.di-Querschnittsbereichen teil als aus den Fachbereichen. Generell sei der Austausch zwischen den Querschnittsabteilungen rege, Angebote der Querschnittsbereiche würden jedoch nicht immer optimal von den Fachbereichen genutzt (Interview 09). Von der eher geringen Beteiligung von FachbereichsvertreterInnen ist nicht nur die Diskussionsgruppe Europa betroffen, sondern auch weitere Diskussions- oder Informationsangebote zu fachbereichsübergreifenden (europäischen) Themen seitens der Querschnittsbereiche (Interviews 03, 09). Die sich hier zeigende Autonomie der Fachbereiche, das Ausmaß und die Art ihres europäischen Engagements selbst zu bestimmen, wird seitens des EU-Verbindungsbüros respektiert (Interview 04).

Obwohl die personelle Ausstattung des EU-Verbindungsbüros von einigen ver.diVertreterInnen (auch außerhalb des Verbindungsbüros) für nicht ausreichend und die Arbeitsbelastung für hoch gehalten wird, wird von vielen dennoch die Reduktion des Bereichs „Europa und Internationales“ auf das EU-Verbindungsbüro aufgrund der allgemeinen Ressourcenknappheit von ver.di und dem damit verbundenen Zwang zur Prioritätensetzung akzeptiert. In den ersten 10 Jahren seit ihrer Gründung verlor ver.di ein Viertel ihrer Mitglieder, und es erfolgte ein massiver Personalabbau von anfangs über 5000 hauptamtlich Beschäftigten auf 3500 in 2011 (Kempe 2011, S. 98). Zwar werde „Europa“ auch als wichtiges Thema gesehen, „das wichtigste Geschäft" seien aber die Tarifverhandlungen. Dafür müsste erst einmal genug Personal vorhanden sein, bevor weitere Stellen für Bereiche wie „Europa“ durchsetzbar seien (Interview 01). ${ }^{7}$ Ferner teilt auch ein/e VertreterIn des Verbindungsbüros die Auffassung einiger Fachbereiche, dass eine Beschäftigung mit „Europa“ im Angesicht konkreter Problemlagen im nationalen bzw. betrieblichen Rahmen schlecht vor den Mitgliedern zu rechtfertigen sei (Interview 04). Europa sei außerdem als politisches Thema nicht Gegenstand von Tarifverhandlungen, sondern politischer Kampagnen, über die man keine Mitglieder gewinne. „Da muss man einfach dann auch immer von der Organisationslogik gucken, wie viel Zeit kann man Europa widmen, wenn man daraus keine Mitglieder gewinnt.“ (Interview 01)

Dem EU-Verbindungsbüro fehlt also mit dem „Mitgliederzugriff“ eine Kapitalsorte, der in ver.di hohe Bedeutung beigemessen wird. Das EU-Verbindungsbüro verfügt über ein relativ knappes Budget und hat mit der Entwicklung aus der Abteilung „Europa und Internationales“ heraus eine Historie der Mittelkürzung. Innerhalb der Organisation ver.di als Feld nimmt das EU-Verbindungsbüro (ähnlich wie andere Querschnittsbereiche auch) eine eher dominierte Position ein. Dies scheint nach meinem bisherigen Eindruck durch andere Kapitalsorten derzeit nicht kompensiert

\footnotetext{
7 Für den Bereich der EBR-Betreuung kommen auch Rüb und Platzer (2015, S. 180) zu dem Befund, dass diejenigen in ver.di, die für die EBR-Arbeit zuständig sind, aufgrund der hohen Auslastung durch das operative Geschäft auf nationaler Ebene wenig Zeit für die EBR-Arbeit aufbringen können.
} 
werden zu können. So verfügen die SekretärInnen des Verbindungsbüros zum Beispiel über ein hohes Sozialkapital in Form von Kontakten zu Abgeordneten des EP, KollegInnen aus den europäischen Gewerkschaftsverbänden und anderen europäischen Gewerkschaften. Dieses ,,in Brüssel“" akkumulierte Sozialkapital scheint allerdings in ver.di-als-Feld kaum nutzbar zu sein. Auch das ,formale“ Kapital, über das das EU-Verbindungsbüro durch seine Ansiedlung im Ressort des ver.di-Bundesvorsitzenden verfügt, gleicht offenbar den Mangel an anderem feldspezifischen Kapital nicht aus.

\section{Schluss}

Insgesamt lässt sich in ver.di keine „Gesamtorganisationsdiskussion zu Europa“ (Interview 04) beobachten. Als gemeinsamer „Rahmen, der für ganz ver.di gilt“ (Interview 04) und innerhalb dessen sich die Europapolitik bewegen sollte, gibt es das ,Europamanifest“ von 2010, ver.dis europapolitische Grundsatzerklärung. Allerdings ist manchen Personen, die sich in ver.di mit Europa beschäftigen, die Existenz dieses Manifests nicht bekannt. Die hohe Ausdifferenzierung von ver.di verhindert nach Einschätzung eines/-r FachbereichsvertreterIn eine koordinierte, kontinuierliche und intensive Beschäftigung mit europäischen Themen und erschwert so die Mitarbeit in europäischen Zusammenhängen (Interview 11). Die einzelnen Fachbereiche in ver.di haben allgemein wenig Berührungspunkte untereinander in ihrer täglichen Arbeit und bekommen auch wenig von der Europapolitik der anderen Fachbereiche mit (Interview 06). Eine engere Koordination der Aktivitäten könnte allerdings notwendig sein, um die Durchsetzungsfähigkeit auf europäischer Ebene zu erhöhen. Keller stellt fest, dass , die Umsetzung von ,Organisationsgröße ‘ in Verhandlungs- und Einflussstärke (...) auf europäischer Ebene keinesfalls automatisch“ erfolgt (Keller 2004, S. 182), sondern erheblichen Koordinationsaufwand sowohl horizontal zwischen den ver.di-Fachbereichen und Fachgruppen sowie vertikal zwischen Fachbereichen und „Gesamtorganisation“ erfordert (Keller 2004, S. 182). Damit eine solche Koordination gelinge, hält Keller einen deutlichen Ausbau der internationalen Abteilungen (nicht nur in ver.di, sondern generell in den nationalen Gewerkschaften) für dringend nötig. Geschehe dies nicht, sei - um auf die Ausgangsfrage dieses Beitrags zurückzukommen - ,ein Teil des Dilemmas vor allem auf europäischer Ebene selbstverschuldet und von den nationalen Gewerkschaften zu verantworten“ (Keller 2004, S. 183).

Aus einer feldtheoretischen Betrachtungsweise, die in diesem Beitrag nur angedeutet werden konnte, lässt sich hinzufügen, dass es nicht allein auf die personelle Ausstattung der Europaabteilungen ankommt, sondern auch auf deren Ausstattung mit feldspezifischem Kapital, d. h. auf ihre Position in der Organisationsstruktur. Derzeit scheint dem „Mitgliederzugriff“ als feldspezifischem Kapital in ver.di eine große Bedeutung zuzukommen. Durch die Festlegungen, die in ver.dis Satzung getroffen wurden, und durch die dominierenden Vorstellungen darüber, wie Mitglieder gewonnen werden, kommt den Fachbereichen in ver.di bei Betrachtung der Verteilung der Kapitalsorte „Mitgliederzugriff“ eine machtvollere Position zu als den Querschnittsbereichen. Ob künftig andere Kapitalsorten in der Organisation an Be- 
deutung gewinnen, die anderen AkteurInnen einen stärkeren Einfluss auf die Gestaltung der Organisationspolitik eröffnen (wie beispielsweise eine stärkere Rolle des EU-Verbindungsbüros bei der Koordinierung von ver.dis Europapolitik), ist abhängig von Auseinandersetzungen und Aushandlungsprozessen innerhalb der Organisation als Feld.

Open access funding provided by Johannes Kepler University Linz.

Open Access Dieser Artikel wird unter der Creative Commons Namensnennung 4.0 International Lizenz (http://creativecommons.org/licenses/by/4.0/deed.de) veröffentlicht, welche die Nutzung, Vervielfältigung, Bearbeitung, Verbreitung und Wiedergabe in jeglichem Medium und Format erlaubt, sofern Sie den/die ursprünglichen Autor(en) und die Quelle ordnungsgemäß nennen, einen Link zur Creative Commons Lizenz beifügen und angeben, ob Änderungen vorgenommen wurden.

\section{Literatur}

Anner, Mark, Ian Greer, Marco Hauptmeier, Nathan Lillie, und Nik Winchester. 2006. The industrial determinants of transnational solidarity: Global Interunion politics in three sectors. European Journal of Industrial Relations 12:7-27. doi:10.1177/0959680106061368.

Bernaciak, Magdalena. 2013. Labour solidarity in crisis? Lessons from general motors. Industrial Relations Journal 44:139-153. doi:10.1111/irj.12009.

Bourdieu, Pierre. 2005. The social structures of the economy. Cambridge/Malden: Polity Press.

Bourdieu, Pierre, und Loïc J.D. Wacquant. 1996. Reflexive Anthropologie. Frankfurt am Main: Suhrkamp.

Dederichs, Andrea M., und Michael Florian. 2002. Felder, Organisationen und Akteure - eine organisationssoziologische Skizze. In Bourdieus Theorie der Praxis. Erklärungskraft - Anwendung - Perspektiven, Hrsg. Jörg Ebrecht, Frank Hillebrandt, 69-96. Wiesbaden: VS Verlag für Sozialwissenschaften.

Emirbayer, Mustafa, und Victoria Johnson. 2008. Bourdieu and organizational analysis. Theory and Society 31:1-44. doi:10.1007/s11186-007-9052-y.

Greer, Ian, und Marco Hauptmeier. 2012. Identity work: Sustaining transnational collective action at General Motors Europe. Industrial Relations 51:275-297. doi:10.1111/j.1468-232X.2012.00677.x.

Hofbauer, Johanna. 2012. Neue Geschlechterordnungen an Hochschulen? Zur theoretischen Fundierung einer empirischen Untersuchung im Sinne der Bourdieu'schen Feldtheorie. In Feldanalyse als Forschungsprogramm I, Hrsg. Stefan Bernhard, Christian Schmidt-Wellenburg, 427-451. Wiesbaden: VS Verlag für Sozialwissenschaften.

Keller, Berndt. 2004. Multibranchengewerkschaft als Erfolgsmodell? Zusammenschlüsse als organisatorisches Novum - das Beispiel ver.di. Hamburg: VSA.

Kempe, Martin. 2011. 10 Jahre ver.di - Die Chancengewerkschaft. Ein Essay. Münster: Westfälisches Dampfboot.

Pernicka, Susanne, und Vera Glassner. 2014. Transnational trade union strategies towards European wage policy: A neoinstitutional framework. European Journal of Industrial Relations 20:317-334. doi:10. 1177/0959680113518232.

Rüb, Stefan. 2009. Die Transnationalisierung der Gewerkschaften. Eine empirische Untersuchung am Beispiel der IG Metall. Berlin: edition sigma.

Rüb, Stefan, und Hans-Wolfgang Platzer. 2015. Europäisierung der Arbeitsbeziehungen im Dienstleistungssektor. Empirische Befunde, Probleme und Perspektiven eines heterogenen Feldes. Berlin: edition sigma.

Vaughan, Diane. 2008. Bourdieu and organizations: The empirical challenge. Theory and Society 37:65-81. doi:10.1007/s11186-007-9056-7.

Nele Dittmar studierte Sozialwissenschaften an der Carl-von-Ossietzky-Universität Oldenburg. Sie ist derzeit Mitarbeiterin im DFG-Projekt „Arbeitsbeziehungen in Europa“ und Dissertantin an der Abteilung für Wirtschafts- und Organisationssoziologie der Johannes Kepler Universität Linz. Ihre Arbeitsschwerpunkte sind Industrielle Beziehungen und die Europäisierung und Transnationalisierung von Gewerkschaften. 